\title{
Searching for very low-mass stars and brown dwarfs with DENIS ${ }^{\star, \star \star}$
}

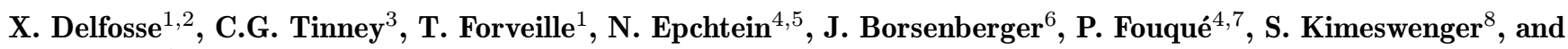 \\ D. Tiphène ${ }^{4}$ \\ 1 Observatoire de Grenoble, 414 rue de la Piscine, Domaine Universitaire de $\mathrm{S}^{\mathrm{t}}$ Martin d'Hères, F-38041 Grenoble, France \\ 2 Observatoire de Genève, CH-1290 Sauverny, Switzerland \\ 3 Anglo-Australian Observatory, PO Box 296, Epping. N.S.W. 2121, Australia \\ 4 DESPA, Observatoire de Paris, 5 place J. Janssen, F-92195 Meudon Cedex, France \\ 5 Observatoire de Nice, BP. 4229, 06304 Nice Cedex 4, France \\ ${ }^{6}$ Institut d'Astrophysique de Paris, 98 bis, Bd. Arago, F-75014 Paris, France \\ 7 European Southern Observatory, Casilla 19001, Santiago 19, Chile \\ 8 Institut für Astronomie der Universität Innsbruck, Technikerstr. 25, A-6020 Innsbruck, Austria
}

Received June 2; accepted September 23, 1998

\begin{abstract}
We present the results of infrared spectroscopic observations of a sample of very low-mass stars and brown dwarf candidates detected in a 230 square degree area by the DENIS (DEep Near Infrared Southern sky; Epchtein, 1997) survey. We find that objects as faint as the faintest known stars are easily detected by DENIS. This sample also includes three members of the new "L" dwarf class, one of which was the first confirmed isolated field brown dwarf. As this data represents $\sim 1 \%$ of the total DENIS survey area, the completed survey can be expected to have a dramatic impact on the study of the faintest stars and brown dwarfs. In particular, it should detect $\sim 300$ of the new and poorly understood "L" class of dwarfs.
\end{abstract}

Key words: stars: low-mass, brown dwarfs - stars: luminosity function, mass function - infrared: stars surveys

\section{Introduction}

The DEep Near-Infrared Survey (DENIS) will be a complete near infrared survey of the southern sky (Epchtein et al. 1994; Epchtein 1997). It aims to provide full coverage in two near-infrared bands $\left(J\right.$ and $\left.K_{\mathrm{s}}\right)$ and one optical band $(I)$, using a ground-based telescope and digital array

Send offprint requests to: X. Delfosse,

e-mail: Xavier.Delfosse@obs.ujf-grenoble.fr

* Based on observations made at the Anglo-Australian Telescope, Siding Spring.

** Figures 2 to 5 are only available at the http://www. edpsciences.com detectors. The approximate $3-\sigma$ limits of the survey are $I=18, J=16, K=13.5$. The products of this survey will be calibrated images and catalogs of point sources and extended sources. The survey started in January 1996 and is expected to be completed within five years.

Two dedicated data analysis centres (DACs) have been created to process this data. The Paris DAC processes the raw data from the telescope into flattened and cleaned images (Borsenberger 1997). The Leiden DAC will then process these images to extract point source information (Deul et al. 1995), which will be assembled into a database at the Paris DAC.

One area of astronomical research in which such a database will have a particularly profound impact is the study of very low-mass stars (VLMs) and brown dwarfs (BDs). Prior to the current generation of all-sky infrared surveys, the two least luminous star/brown dwarf objects known - Gl229B (Nakajima et al. 1995) and GD165B (Becklin \& Zuckerman 1988) - were both detected as companions to brighter nearby stars. While such "looking for things around other things" techniques improve the chances of finding rare objects, they do not allow us to determine their space density. Younger and warmer free floating brown dwarfs have also been identified in the Pleiades cluster (Rebolo et al. 1995; Basri et al. 1996). The most recent Pleiades work (Zapatero Osorio et al. $1997 ; 1998)$ suggests a mass function which rises into the brown dwarfs regime as $\mathrm{d} N / \mathrm{d} M \sim M^{-1}$ (Martín et al. 1998). VLMs and BDs may be thus a numerous, if not dynamically significant, galactic disk population. The space density of the VLM/BDs is thus of fundamental importance to star formation theory. However, because the initial mass function in a particular young cluster may not be representative of the disk altogether (since segregation 
and cluster evaporation are mass sensitive processes), it is important to determine mass functions for the overall field population.

To date, studies of these populations in the field have been severely hampered by the difficulty of finding such objects. The most sensitive (i.e. largest volume) surveys to date have been carried out using $R$ and $I$ band photographic plates from the Palomar and United Kingdom 48"-class Schmidt telescopes (e.g. Tinney 1992; Hawkins \& Bessell 1988; Gilmore et al. 1985; Reid \& Gilmore 1984). However, for the latest-type objects, such searches are limited by the $R$ plate sensitivity and are only complete to $I \approx 16.5-17$. Typically less than one M9-10 dwarf is found in each $6^{\circ} \times 6^{\circ}$ Schmidt field. With a completeness limit of $I \approx 18$, and coverage of the whole southern sky, DENIS therefore offers the chance to detect many more examples of this poorly understood class of object. Moreover, the optical-infrared $I-J$ and $I-K$ colours provided by DENIS are well suited to selecting very cool stars and brown dwarfs (Allard 1998; Burrows et al. 1997).

DENIS will sample very large volumes. The maximum distance for the detection of GD165B-like objects is $20 \mathrm{pc}$, meaning the space density for such objects can be measured to $3-\sigma$ upper limits of $1.810^{-4} \mathrm{pc}^{-3}$. DENIS is less sensitive to Gl229B-like objects - the limiting distance set by the $I$-band is $2.3 \mathrm{pc}$. However because the two infrared bands are observed simultaneously with the optical, upper limits are significant, increasing the distance limit to $6.3 \mathrm{pc}$, and the $3-\sigma$ upper limit to $5.710^{-3} \mathrm{pc}^{-3}$. A constant extrapolation of the observed low-mass stellar luminosity function would predict that DENIS will detect several hundred GD165B-like objects, a few Gl 229B-like objects, and many objects (and much needed new data) on objects with intermediate luminosities (Kirkpatrick et al. 1997a). In particular, observation of these new brown dwarfs will address issues fundamental to our understanding of very low temperature atmospheres, such as the importance of dust formation (Allard 1998; Jones \& Tsuji 1997; Tsuji et al. 1996a,b), and the importance of previously unrecognized opacity sources like $\mathrm{CH}_{4}$ (Tsuji et al. 1995) and CrH (Kirkpatrick et al. 1998).

However, to address these issues, significant follow-up programs will be required. With \pm 0.3 magnitude photometry at the survey limits, the DENIS catalogues by themselves cannot be used to construct luminosity functions: first, samples of nearby late-type objects will to some extent be contaminated by distant late-type giants; second, as the number of stars in the catalogs is a steep function of colour (i.e. there are many fewer red stars than blue ones), the number of stars in any one colour (or equivalently luminosity) bin, will be contaminated by stars scattered in from bluer bins; and lastly, cosmic rays and intermittent bad pixels can create spuriously red objects if they happen to overlap an otherwise normal star in one of the infrared pass-bands. In other words, the raw catalog of VLM stars and brown dwarfs produced by DENIS will be a "dirty" sample. What is this contamination level, and what type of observations will most efficiently "clean-up" the DENIS catalogue? This is the major issue we seek to address in this paper.

\section{The mini-survey}

We have carried out a "Mini-survey" with spectroscopic follow up on the very low-mass star and brown dwarf candidates contained in $\approx 1 \%$ of the DENIS survey data. The "Mini-survey" data are representative of the survey quality, and its results can therefore be scaled to evaluate the brown dwarf content of DENIS. The image data from the high latitude part $\left(\left|b_{\mathrm{II}}\right|>20-30^{\circ}\right)$ of 47 survey strips (for a total surface area of 230 square degree), as produced by the Paris DAC, were hand processed to create catalogs of $I, J$ and $K^{\prime}$ photometry. From these we identified a sample of objects for which infrared $H$ - and $K$-band spectroscopy was carried out, in order to "clean" the dirty sample and evaluate its level of contamination.

\subsection{The DENIS data}

DENIS observations are carried out on the ESO $1 \mathrm{~m}$ telescope at Cerro La Silla (Chile), with a purpose-built three channel infrared camera (Copet et al. 1998). Dichroic beam splitters separate the three channels, and focal reducing optics provide a $12^{\prime}$ field of view for all the channels. The detectors used give image scales of $3^{\prime \prime} /$ pixel on the $256 \times 256$ NICMOS3 arrays used for the two infrared channels and $1^{\prime \prime} /$ pixel on the $1024 \times 1024$ Tektronix CCD detector of the I channel. A focal plane microscanning mirror is used to obtain $1^{\prime \prime}$ sampling for the two infrared channels. Cold filters define the spectral bandpasses which are close to Gunn $i, J_{\mathrm{CIT}}$, and Mauna Kea $K_{\text {short }}$ (Copet et al. 1998). The sky is scanned in a step and stare mode, along 30 degrees strips at constant right ascension. These strips are centered at $\delta=$ $-13^{\circ},-43^{\circ} \&-73^{\circ}$, and are the basic DENIS observing unit. Photometric standards are observed at the beginning and end of each strip. Strip spacing and image spacing within a strip are both $10^{\prime}$, providing $20 \%$ overlap in right ascension. Integration time is $\sim 10 \mathrm{~s}$.

The 47 strips used for the Mini-survey (see Table 1) were chosen to maximise galactic latitude and to obtain useful right ascension coverage during our follow-up spectroscopic observing runs - the table is split into two sections as slightly different selection criteria were used for the northern and southern Galactic hemisphere samples. The image data were obtained from the Paris DAC and had been processed with version 4.2 of the standard pipeline software (Borsenberger 1997). The background levels are derived from a local clipped mean along the strip. Flat-field corrections are derived using observations of the dawn sky. 


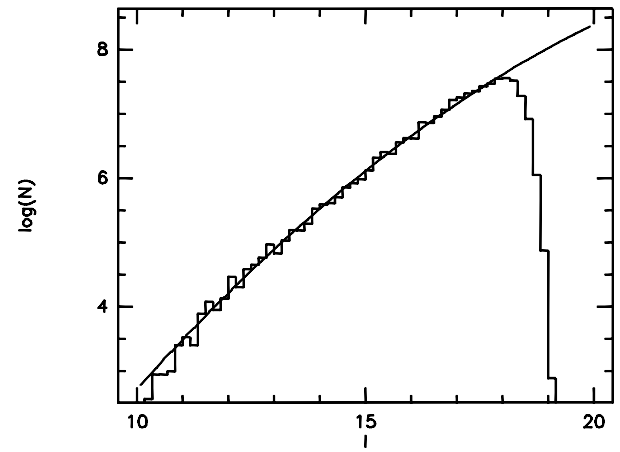

Strip 3128
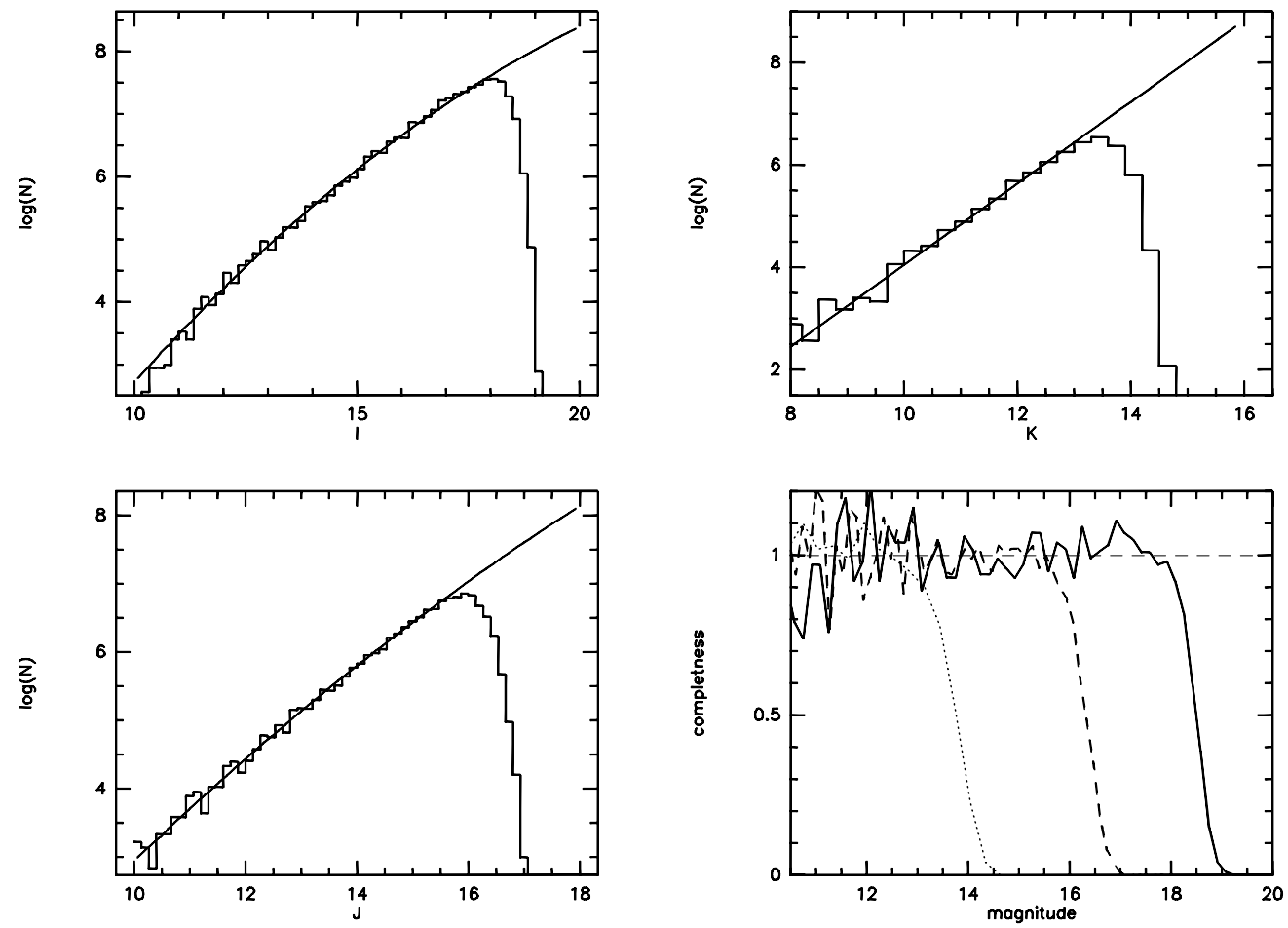

Fig. 1. Completeness plot for Strip 3128

When this project was commenced the Leiden source extraction pipeline was not yet operational. Source detection and photometry was therefore performed in Grenoble, using the SExtractor package (Bertin \& Arnouts 1996). Sources were detected after smoothing the image with a $2^{\prime \prime}$ FWHP kernel, requesting a minimum of 5 contiguous pixels above a threshold of 1.5 standard deviations of the original image for the southern sample and 2.5 for the northern one. Adaptive aperture photometry was then extracted from the original unsmoothed image. The images of the closest photometric standards were identically processed, and used to define the zero point of the instrumental magnitudes. Since the objects of interest in our survey are close to our limiting magnitude, their photometric uncertainties are significant. As a result, we have not attempted to apply the small corrections appropriate to the airmass difference between our standard and strip observations, nor did we correct for colour terms.

Completeness curves were estimated for each strip by fitting low order polynomials $(n=1-3)$ to the brighter part of the differential number count (i.e. $\log (N) / \log (S)$ ) curve for that strip, and using this fit to normalise the $\log (N) / \log (S)$ curve. This normalised $\log (N) / \log (S)$ curve was then used to evaluate the magnitude at which the strip is $50 \%$ incomplete. A typical example is shown in Fig. 1 and the resulting completeness limits for each strip are listed in Table 1. The variation in completeness level reflects changes in the sky level - due to the moon phase for $I$, and to ambient temperature variations for $K$.

\subsection{Spectroscopic sample selection}

Even though VLMs and brown dwarfs are brightest at near-infrared wavelengths, they are still vastly outnumbered in our flux limited samples by intrinsically brighter and more distant stars. As an illustration, the present mini-survey has obtained photometry at $I$ and $J$ for some $610^{5}$ stellar objects, of which less than 50 were finally retained as VLM/BD candidates. With such a low selection fraction it is essential that the selection scheme has a very low false alarm rate, since even a small "blue leak" of $0.01 \%$ would still produce a sample dominated by $\mathrm{G}$ and $\mathrm{K}$ dwarfs.

Contamination by distant disk red giants increases at low galactic latitudes, as does the general stellar background within which we have to search for the brown dwarf signal. We initially imposed a galactic latitude limit of $\left|b_{\mathrm{II}}\right|>30^{\circ}$ - though this was later relaxed to $\left|b_{\mathrm{II}}\right|>20^{\circ}$ as our confidence in the processing increased. All objects in $650^{\prime} \times 550^{\prime}$ and $280^{\prime} \times 160^{\prime}$ zones centered on the LMC $\left(5^{\mathrm{h}} 23.6^{\mathrm{m}}-69^{\circ} 45^{\prime}\right)$ and the SMC $\left(0^{\mathrm{h}} 52.7^{\mathrm{m}}-72^{\circ} 50^{\prime}\right)$ were also rejected, to avoid contamination by cool Magellanic Cloud giants.

Objects detected within $20^{\prime \prime}$ of the edge of an image were ignored, as were objects with clearly non 
Table 1. Mini-survey strips with $50 \%$ completeness limits Strip $\alpha(2000) \quad \delta(2000)^{a}$ Epoch Used $I_{\lim } \quad J_{\lim } \quad K_{\lim }$

(a) Northern Galactic Pole Mini-survey strips

$\begin{array}{llllllll}3296 & 08: 59: 19 & -13^{\circ} & 1996.0 & 2.11^{\circ} & 17.89 & 16.02 & 13.18 \\ 3382 & 09: 09: 19 & -13^{\circ} & 1996.0 & 3.59^{\circ} & 18.23 & 16.11 & 13.47 \\ 3329 & 09: 10: 00 & -13^{\circ} & 1996.0 & 3.52^{\circ} & 18.38 & 16.17 & 13.57 \\ 3403 & 09: 12: 39 & -13^{\circ} & 1996.0 & 3.27^{\circ} & 18.35 & 16.22 & 13.54 \\ 3444 & 09: 40: 00 & -13^{\circ} & 1996.1 & 5.41^{\circ} & 17.51 & 16.02 & 13.48 \\ 3347 & 09: 44: 40 & -13^{\circ} & 1996.0 & 5.35^{\circ} & 18.32 & 16.14 & 13.45 \\ 3317 & 09: 45: 59 & -13^{\circ} & 1996.0 & 5.35^{\circ} & 18.11 & 16.17 & 13.30 \\ 3362 & 10: 05: 19 & -13^{\circ} & 1996.0 & 5.48^{\circ} & 18.32 & 16.23 & 13.45 \\ 3415 & 10: 07: 59 & -13^{\circ} & 1996.0 & 5.63^{\circ} & 18.07 & 16.14 & 13.51 \\ 3383 & 10: 25: 19 & -13^{\circ} & 1996.0 & 5.32^{\circ} & 18.05 & 16.08 & 13.48 \\ 3330 & 10: 26: 00 & -13^{\circ} & 1996.0 & 5.51^{\circ} & 18.71 & 16.64 & 13.78 \\ 3312 & 10: 31: 19 & -13^{\circ} & 1996.0 & 5.67^{\circ} & 18.23 & 15.99 & 13.09 \\ 3445 & 10: 58: 40 & -13^{\circ} & 1996.1 & 5.54^{\circ} & 18.04 & 16.47 & 13.81 \\ 3405 & 11: 44: 00 & -13^{\circ} & 1996.0 & 2.90^{\circ} & 18.29 & 16.38 & 13.42 \\ 3416 & 11: 54: 40 & -13^{\circ} & 1996.0 & 5.60^{\circ} & 18.26 & 16.17 & 13.48 \\ 3446 & 12: 27: 59 & -13^{\circ} & 1996.1 & 5.63^{\circ} & 17.84 & 16.20 & 13.42\end{array}$

(b) Southern Galactic Pole Mini-survey strips

$\begin{array}{llllllll}4386 & 00: 21: 32 & -43^{\circ} & 1996.7 & 5.63^{\circ} & 18.47 & 16.20 & 13.96 \\ 4395 & 00: 46: 09 & -43^{\circ} & 1996.7 & 5.63^{\circ} & 18.41 & 16.35 & 14.05 \\ 4387 & 01: 42: 18 & -43^{\circ} & 1996.7 & 3.84^{\circ} & 18.50 & 16.17 & 13.93 \\ 3127 & 01: 50: 39 & -73^{\circ} & 1995.9 & 1.57^{\circ} & 18.60 & 16.26 & 13.90 \\ 3135 & 02: 05: 19 & -13^{\circ} & 1995.9 & 5.60^{\circ} & 18.42 & 16.14 & 13.60 \\ 4396 & 02: 05: 23 & -43^{\circ} & 1996.7 & 5.63^{\circ} & 18.47 & 16.29 & 14.02 \\ 3146 & 02: 33: 59 & -13^{\circ} & 1995.9 & 5.60^{\circ} & 18.60 & 16.44 & 13.48 \\ 3171 & 02: 47: 59 & -13^{\circ} & 1995.9 & 5.10^{\circ} & 18.56 & 16.50 & 13.35 \\ 3111 & 03: 03: 59 & -73^{\circ} & 1995.9 & 5.60^{\circ} & 18.28 & 16.14 & 13.78 \\ 3128 & 03: 09: 19 & -73^{\circ} & 1995.9 & 5.54^{\circ} & 18.49 & 16.32 & 13.78 \\ 3136 & 03: 31: 19 & -13^{\circ} & 1995.9 & 5.67^{\circ} & 18.56 & 16.32 & 13.57 \\ 3147 & 03: 52: 40 & -13^{\circ} & 1995.9 & 5.67^{\circ} & 18.57 & 16.38 & 13.45 \\ 3154 & 03: 56: 09 & -43^{\circ} & 1995.9 & 1.04^{\circ} & 18.65 & 16.68 & 14.14 \\ 3172 & 04: 05: 19 & -13^{\circ} & 1995.9 & 5.26^{\circ} & 18.71 & 16.59 & 13.06 \\ 3339 & 04: 26: 39 & -73^{\circ} & 1996.0 & 3.46^{\circ} & 18.59 & 16.35 & 13.72 \\ 3129 & 04: 29: 20 & -73^{\circ} & 1995.9 & 3.77^{\circ} & 18.53 & 16.20 & 13.69 \\ 3137 & 04: 49: 20 & -13^{\circ} & 1995.9 & 5.67^{\circ} & 18.74 & 16.53 & 13.48 \\ 3340 & 05: 41: 20 & -73^{\circ} & 1996.0 & 3.68^{\circ} & 18.50 & 16.38 & 13.72 \\ 3113 & 05: 57: 20 & -73^{\circ} & 1995.9 & 3.62^{\circ} & 18.42 & 16.20 & 13.57 \\ 4083 & 20: 26: 55 & -43^{\circ} & 1996.4 & 5.67^{\circ} & 18.26 & 16.35 & 13.66 \\ 4103 & 20: 31: 32 & -43^{\circ} & 1996.4 & 5.67^{\circ} & 18.14 & 16.41 & 13.78 \\ 4196 & 20: 39: 59 & -43^{\circ} & 1996.5 & 5.63^{\circ} & 18.47 & 16.44 & 13.84 \\ 4101 & 20: 39: 13 & -43^{\circ} & 1996.4 & 5.67^{\circ} & 18.41 & 16.23 & 13.63 \\ 4091 & 20: 42: 18 & -43^{\circ} & 1996.4 & 5.67^{\circ} & 18.55 & 16.50 & 13.78 \\ 4383 & 20: 52: 18 & -43^{\circ} & 1996.7 & 5.67^{\circ} & 18.62 & 16.50 & 13.93 \\ 4204 & 21: 35: 20 & -13^{\circ} & 1996.5 & 5.67^{\circ} & 18.50 & 16.32 & 13.48 \\ 4402 & 21: 45: 59 & -13^{\circ} & 1996.7 & 5.67^{\circ} & 18.35 & 16.50 & 13.84 \\ 4192 & 21: 46: 39 & -73^{\circ} & 1996.5 & 5.67^{\circ} & 17.93 & 16.05 & 13.60 \\ 4197 & 22: 33: 04 & -43^{\circ} & 1996.5 & 5.63^{\circ} & 18.56 & 16.38 & 13.96 \\ 4385 & 23: 03: 50 & -43^{\circ} & 1996.7 & 5.67^{\circ} & 18.56 & 16.47 & 13.90 \\ 4403 & 23: 04: 40 & -13^{\circ} & 1996.7 & 5.63^{\circ} & 18.38 & 16.65 & 13.90\end{array}$

Notes: $a$ - Strips centered on: $-13^{\circ} \operatorname{span} \delta=+2^{\circ}$ to $-28^{\circ}$; $-43^{\circ} \operatorname{span} \delta=-28^{\circ}$ to $-58^{\circ} ;-73^{\circ}$ span $\delta=-58^{\circ}$ to $-88^{\circ}$. stellar parameters. At the relatively bright fluxes sampled by DENIS, galaxies are rare and typically much bluer than late $\mathrm{M}$ dwarfs, and would therefore have been eliminated by colour alone. Morphological rejection was primarily implemented to exclude cosmic rays and some optical ghosts. Finally, all sources within a $6 \times 6$ pixel zone 86 pixels north of every bright $(J<10, K<8)$ source were ignored. Multiple reflections within the DENIS dichroic splitters produce a faint infrared ghost, which has no counterpart at $I$ and can thus have extremely red apparent colours.

The three individual channels were then merged, using the $I$ channel as a position reference. A linear transformation (offset, rotation, and scaling factor) between the other channels and this master channel was determined by minimizing the sum of the squared distances between all unsaturated stars brighter than $I<17, J<15$ and $K<13.4$. The $J$ and $K$ object lists were then searched for matching objects within $3^{\prime \prime}$ of each $I$ object to produce a three colour catalog for each strip. Because source confusion is never a problem at the galactic latitudes analysed here, this simple procedure was extremely effective.

Candidates were then selected in the three colour catalogs. To avoid contamination by cosmic rays, sources were required to be detected in at least two pass bands. For the objects of interest, $J$ is always the most sensitive passband, so two classes of objects were selected: (1) objects with a very red $I-J$; and (2) objects with $J$ and $K$ detections but no $I$ detection. The latter criterion aimed to select extremely low temperature objects $\left(T_{\text {eff }} \approx 1000 \mathrm{~K}\right)$ with colours similar to Gl $229 \mathrm{~B}$ - visual inspection of all these candidates revealed no reliable detections beyond objects also selected on $I-J$. In the remainder of this paper we consider only objects with very red $I-J$ colours. The northern sub-sample was processed first, and we selected all objects with $I-J>2.75$, or $I-J>2.2$ for the brighter ones. In view of the large number of selected objects with large photometric errorbars (and presumably with an actual $I-J$ bluer than 2.5), we changed the selection criteria for the southern sample and retained all objects with $(I-J)-\sigma(I-J)>2.5$.

Inspection of the image data for these selected objects showed that $\sim 80 \%$ were contaminated by bad pixels or cosmic rays, and had much bluer intrinsic colours. This large artefact fraction illustrates a well known difficulty when looking for needles in haystacks (the population of interest represents less than $0.01 \%$ of the number of detected stars). It suffices that a very small fraction of the $99.99 \%$ of higher mass stars is affected by a bad pixel or a cosmic rays for it to dominate the very low mass star and brown dwarfs region of the color-color diagram. Since such contamination could not be selected against using the extracted source parameters, we visually inspected the image data for all the initially selected VLM/BD candidates. One reason for this relatively high level of sample contamination is that version 4.2 of the Paris DAC software used incorrect bad pixel flagging - future DENIS 
data will be significantly improved in this respect. Cosmic rays will also be identified with increased precision in future DENIS data by a neural network classifier, to be used in the next generation of the Leiden DAC extraction software (E. Bertin, private communication).

The objects remaining after this visual sample culling are listed in Table 2. Table 2a shows the list of objects identified for spectroscopic follow-up which constitute a sample with $I-J>2.8$. Table $2 \mathrm{~b}$ lists the remaining objects selected from the DENIS data. The positions provided are based on the telescope encoder readings, and are accurate to $\pm 10-20^{\prime \prime}$, Much better position will be produced by the final DENIS pipeline. The listed photometric errors are internal errors determined by the photometry package, and will thus be underestimates for the brighter stars. Figure 6 presents the selected objects detected in all three colours in a colour-colour diagram, $I-J$ versus $J-K$.

\subsection{Infrared spectroscopy}

Infrared spectroscopic observations were carried out on the $3.9 \mathrm{~m}$ Anglo-Australian Telescope (AAT) on the nights of 1996 April 9 and 10 (UT) and 1996 October 21 and 22 (UT). On both runs the Infra-Red Imaging Spectrograph (IRIS - Allen et al. 1993) was used in its cross-dispersed HK echelle mode. This provides complete wavelength coverage from $1.438-2.536 \mu \mathrm{m}$, at a resolution of $\lambda / \Delta \lambda=$ 440 , and a dispersion of $\lambda / \Delta \lambda=780$. A slit of width $1.4^{\prime \prime}$ and length $13^{\prime \prime}$ was used.

Observations were typically of 20 minutes total integration time, and were made with the object being nodded between two positions on the slit. Reductions were performed using the Figaro data reduction package (Shortridge 1993) and followed a standard procedure: the data were sky subtracted using pairs of nodded observations, straightened to remove the curvature of the echelle orders and the wavelength dependent "tilt" of the IRIS slit, and extracted using a modified version of the Figaro ANAL routine to remove any residual sky spectrum left after pair-subtraction. A variety of arcs ( $\mathrm{Ne}, \mathrm{Ar}, \mathrm{Cu}, \mathrm{Hg}$ and $\mathrm{Xe}$ ) were used to construct a wavelength calibration good to $\pm 2.5 \AA$, which was applied to all the spectra.

Spectra of late F-type and early G-type stars were used to create flux calibrations. Because of the water vapour content at the AAT site, we did not attempt to correct for absorption near the atmospheric $\mathrm{H}_{2} \mathrm{O}$ bands. Standards were observed every few hours, at airmasses within \pm 0.2 of the program object observations. The observed standards had their H Brackett lines corrected by hand. The lines were identified by dividing each standard by a G-type spectrum in which the $\mathrm{H}$ lines are negligibly weak. The $\mathrm{CO}$ bands beyond $2.2 \mu \mathrm{m}$ were not corrected, as these were weak (i.e. less than a few percent) in even the latest G5 standards. Lastly, the photometry of Carter \& Meadows (1995) was used to put these standards on an approximate flux scale. While the relative fluxes obtained for our program stars are good to better than $5 \%$, the absolute fluxes are no better than $\pm 30 \%$.

Figures 7 and 8 present a sample of the spectra obtained for the program objects listed in Table 2. A sample of comparison objects was also observed - in particular four late-type giants, and a large number of late-type dwarfs. These are listed in Table 3 and shown in Figs. 9 and 10. Because the AAT is a relatively low-altitude site, it is not possible to make observations through the atmospheric water vapour bands. These have been marked on the figures. However, even outside these regions both the dwarf and giant spectra show the broad stellar $\mathrm{H}_{2} \mathrm{O}$ absorption bands characteristic of these low temperature atmospheres. CO bandheads are seen from $2.3-2.4 \mu \mathrm{m}$ in all the spectra, though some of the giant spectra also show $\mathrm{CO}$ in the $1.6-1.7 \mu \mathrm{m}$ region. Numerous spectral lines due to neutral metals are also seen - in particular, Na I $\lambda 2.20 \mu \mathrm{m}$ and Ca I $\lambda 1.614 \mu \mathrm{m}$ (Tinney et al. 1993). There is also a strong absorption in many of the dwarfs at $\lambda 1.627 \mu \mathrm{m}$, which remains unidentified.

\subsection{Infrared spectral classification}

\subsubsection{Giant/dwarf discrimination}

A comparison of the giants and dwarfs in Figs. 9 and 10 shows that for high signal-to-noise ratio observations the presence of $\mathrm{Na}$ in absorption at $2.20 \mu \mathrm{m}$ indicates that the atmosphere is at high (i.e. dwarf) gravities (Jones et al. 1994; Tinney et al. 1993). However, for much of our data, such a criteria cannot be used because of the signalto-noise available. A giant dwarf discriminant which can be used at lower $\mathrm{S} / \mathrm{N}$ is the strength of $\mathrm{CO}$ bandhead at $2.29 \mu \mathrm{m}$. Following Jones et al. (1993) we therefore define a $\mathrm{CO}$ index as the ratio of the integrated flux in bands at $(2.22-2.28) \mu \mathrm{m}$ and $(2.30-2.36) \mu \mathrm{m}$.

CO index $=\int_{2.22 \mu \mathrm{m}}^{2.28 \mu \mathrm{m}} F_{\nu} \mathrm{d} \nu / \int_{2.30 \mu \mathrm{m}}^{2.36 \mu \mathrm{m}} F_{\nu} \mathrm{d} \nu$.

Figure 11a shows this $\mathrm{CO}$ index as a function of $I-$ $J$ colour, for the comparison objects we observed. No $I-J$ colours are available for the giants in our comparison sample, and as these objects are all long-period variables, colour information would not necessarily be meaningful. The measured giant ratios are thus shown as arrows at the appropriate CO index level. The expected dependence is seen, in that $\mathrm{CO}$ is much stronger in giants than in dwarfs. We therefore define a criterion that if the $\mathrm{CO}$ index $>1.24$ the object is classified as a giant. This classifies two sources, as giants - DENIS-P J1228-2510 and J0944-1310.

The giant classification of DENIS-P J1228-2510 is supported by its position above the dwarf sequence in Fig. 6 and its bright apparent magnitude $(I=10.7, I-K=3.7)$. 
Table 2. The DENIS Mini-survey Spectroscopic sample

\begin{tabular}{cccccccc}
\hline DENIS-P \# & $\alpha, \delta$ & $I$ & $J$ & $K$ & $I-J$ & $\mathrm{H}_{2} \mathrm{O}$ Index & $\mathrm{CO}^{b}$ \\
& $(2000)^{a}$ & & & "H" & "K" &
\end{tabular}

\begin{tabular}{|c|c|c|c|c|c|c|c|c|c|}
\hline \multicolumn{10}{|c|}{ Sample (a) - Spectroscopically complete for $I-J \geq 2.8$} \\
\hline J0020-4414 & $00: 20: 59.4-44: 14: 43$ & $18.32 \pm 0.16$ & $14.97 \pm 0.06$ & $13.60 \pm 0.15$ & $3.35 \pm 0.17$ & $3.2 \pm 0.4$ & $2.2 \pm 0.3$ & 1.11 & $11.0 \pm 0.5$ \\
\hline J0021-4244 & $00: 21: 05.7-42: 44: 50$ & $16.83 \pm 0.05$ & $13.63 \pm 0.02$ & 6 & $3.20 \pm$ & & & 1.13 & \\
\hline J0142-4715 & $6.0-47: 15: 06$ & $18.40 \pm 0.16$ & $15.54 \pm 0.17$ & - & & & & 05 & \\
\hline-6430 & $9-64:$ & .12 & $14.46 \pm$ & $3 \pm$ & & & & & 工 \\
\hline J0205-0653 & $2-06: 53: 36$ & $16.83 \pm 0.06$ & $13.94 \pm 0.04$ & & & & & & \\
\hline \multirow[t]{2}{*}{ J0205-1159 } & 02:05:29.0 - 11:59:25 & $18.30 \pm 0.24$ & $14.63 \pm 0.06$ & $13.00=$ & & & & & \\
\hline & & & & & & & & 1.23 & \\
\hline-1637 & $3-16$ : & \pm 0.22 & $15.60 \pm$ & - & & & & & \\
\hline J020 & $9-33$ & 08 & 05 & \pm & & & & 12 & -0. \\
\hline J0247-1055 & $8-10:$ & \pm 0.18 & $15.37 \pm 0.06$ & 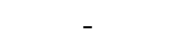 & & & & 9 & $<8$ \\
\hline J0426-5735 & $.9-57: 35: 51$ & $18.45 \pm 0.20$ & $15.28 \pm$ & - & & & 1.0 & & $<8$ \\
\hline J0429-6649 & $6-66: 49: 57$ & $17.32 \pm 0.13$ & $14.43 \pm 0.05$ & $0 \pm$ & & & & 5 & 0.5 \\
\hline J0430-8314 & $0-83: 14: 57$ & $18.33 \pm 0.23$ & $15.02 \pm 0.18$ &  & & & & & \\
\hline-0228 & $3-02: 28: 35$ & \pm 0.10 & $14.50 \pm$ & 2 & & & & & \\
\hline-2225 & $1-22: 25: 02$ & \pm 0.20 & $15.86 \pm$ & - & & & & & 1.5 \\
\hline-8433 & $0-84: 3$ & \pm 0.19 & 15.17 & - & & & & & $<8$ \\
\hline J0909-0658 & $1-06: 5$ & $17.21 \pm 0.08$ & $14.01 \pm$ & $1 \pm$ & & & & & 0.5 \\
\hline J0944-1310 & $5-13: 10: 20$ & \pm 0.21 & $15.70 \pm$ & - & & & & 5 & \\
\hline-1706 & $8-17:$ & .14 &  & - & & & & & \\
\hline 637 & $2-06:$ & 11 & 8 & 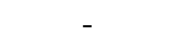 & & & & & \\
\hline 548 & $5-15$ : & 17 & 4 & $1 \pm$ & & & & & \\
\hline $\mathrm{J} 1227+0114$ & $0+01:$ & \pm 0.10 & 14. & & & & & & \\
\hline \multirow[t]{2}{*}{$\mathrm{J} 1228-1547$} & $8-15:$ & & & & & & & & \\
\hline & & & & & & & & & \\
\hline & -2 & & & . & & & & & \\
\hline 45 & -32 & 16 & 7 & - & & & & 5 & \\
\hline 700 & & $=0.17$ & $1-8$ & - & & & & & \\
\hline J214 & $8 \quad 01$ & 1 & 15 & - & & & & & \\
\hline \multicolumn{10}{|c|}{ Sample (b) - Remaining Objects } \\
\hline & & & & - & & & & & $9.6 \pm 1.6$ \\
\hline & $3-43:$ & 17. & 15.1 & - & & & & & $<8$ \\
\hline 0019 & $1+00: 19: 02$ & $18.40 \pm 0.20$ & 15.7 & 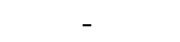 & & 1 & 6 & 1.08 & $<8$ \\
\hline & -0 & 4 & 15. & - & & & - & 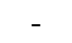 & \\
\hline 14 & -04 & 6 & 14.25 & 1 & & 3 & 2 & 1.15 & $=0.7$ \\
\hline & $8-11: 59: 25$ & \pm 0.08 & $13.85 \pm 0.04$ & $2 \pm$ & & - & - & - & \\
\hline & $1.8-22: 57: 49$ & $17.02 \pm 0.10$ & $14.23 \pm$ & $13.24 \pm$ & & & & 1.14 & $10.9 \pm 0.7$ \\
\hline & $7-09: 00: 28$ & \pm 0.18 & 15.61 & - & & & & 7 & $<8$ \\
\hline & $1-13:($ & \pm 0.05 & 14.1 & 7 & & \pm & 2 & 1.11 & \\
\hline 2 & 12 & 3 & 13.1 & $7 \pm$ & & - & - & 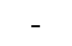 & \\
\hline-1437 & $8: 10.9-14: 37: 38$ & $17.61 \pm 0.12$ & $15.00 \pm 0.06$ & - & & - & - & - & \\
\hline-1956 & $4.8-19: 56: 23$ & $5 \pm 0.03$ & $13.27=$ & 6 & & - & - & - & \\
\hline & $0-15: 09: 55$ & $3 \pm 0.08$ & 14.00 & & & - &  & - & \\
\hline-2410 & $.3-24: 10: 39$ & $16.06 \pm 0.04$ & $13.86 \pm$ & $12.70 \pm$ & & 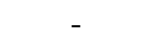 & 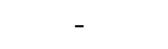 & - & \\
\hline & $11: 54: 27.3-09: 43: 06$ & $15.97 \pm 0.04$ & $13.66 \pm$ & $12.83 \pm$ & & - & - & - & \\
\hline $54+0135$ & $11: 54: 33.9+01: 35: 45$ & $16.03 \pm 0.04$ & $13.26 \pm 0.02$ & $12.17 \pm 0.06$ & $2.77 \pm 0$ & $2.7 \pm 0.2$ & $2.1 \pm 0.1$ & 1.14 & $10.3 \pm 0.6$ \\
\hline $\mathrm{J} 1227+0005$ & $12: 27: 47.6+00: 05: 48$ & $17.80 \pm 0.17$ & $15.05 \pm 0.09$ & - & 2.75 & - & - & - & \\
\hline $\mathrm{J} 1228-2510^{(c)}$ & $12: 28: 05.6-25: 10: 17$ & $10.66 \pm 0.01$ & $8.25 \pm 0.01$ & & & & & 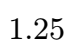 & \\
\hline J2052-5512 & $20: 52: 55.0-55: 12: 03$ & $17.52 \pm 0.13$ & $14.82 \pm 0.07$ & $14.08 \pm$ & & $2.0 \perp$ & & 0.98 & $=2.8$ \\
\hline $\mathrm{J} 2143-8337$ & $21: 43: 41.4-83: 37: 03$ & $16.21 \pm 0.05$ & $13.44 \pm 0.03$ & $12.44 \pm 0.11$ & $2.77 \pm 0.06$ & $2.8 \pm 0.6$ & $2.9 \pm 0.2$ & 1.12 & $10.5 \pm 1.3$ \\
\hline
\end{tabular}

Notes :

$a$ - Positions are given in equinox J2000.0 and have epochs between 1995.9 and 1996.7

$b$ - Uncertaint \pm in CO index $< \pm 0.01$.

$c$ - IR spectrum showed this object is not a late-type star at all. 


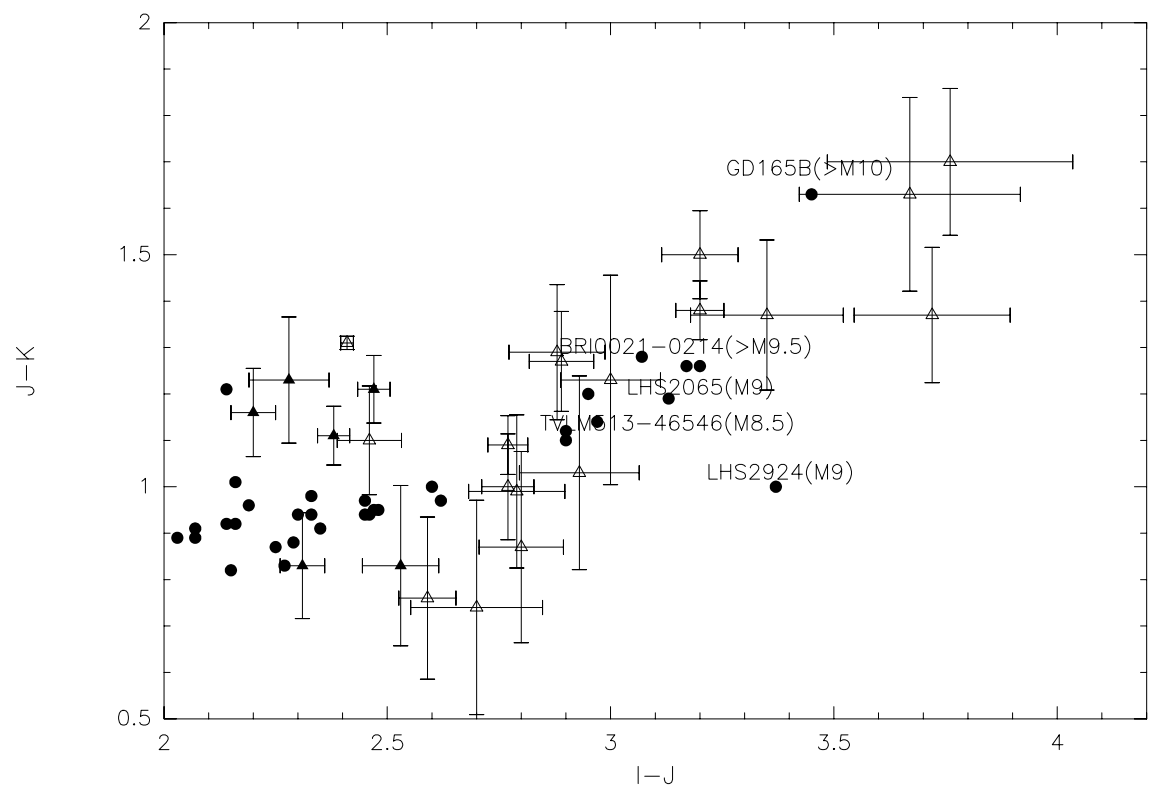

Fig. 6. $I-J / J-K$ colour-colour diagram for the objects selected from the DENIS strips and for templates from the literature. Open triangle: spectroscopically observed; filled triangle: not spectroscopically observed; solid circle: template M dwarfs from Leggett (1992) and Tinney, Mould \& Reid (1993). All DENIS objects redder than our completeness limit of $I-J=2.8$ were spectroscopically observed

If we assume a dwarf status for this star the colourmagnitude diagram of Tinney (1996) would put it at a distance of $\approx 4$ pc. Discovering such a nearby star in the limited area covered by the present survey is unlikely. Giant stars at these effective temperatures have $M_{\text {bol }} \sim-2.9$ (Lang 1991), or $M_{K} \sim-6.0$ (Bessell et al. 1998). If we interpret DENIS-P J1228-2510 and J0944-1310 as being giants then, we place them at distances of $\sim 4 \mathrm{kpc}$ and $\sim 125 \mathrm{kpc}$ respectively. The latter is extreme for giant stars, but not unreasonably so. Carbon stars, for example, are known at distances of up to $\sim 100 \mathrm{kpc}$ (Totten \& Irwin 1998). The giant status of J0944-1310 is, however, based on one of our noisier infrared spectra and will require confirmation.

DENIS-P J1228-2510 is bright enough that its DENIS colours are well determined, and they show that it lies 0.4 magnitudes above the dwarf sequence in the $I-J / J-$ $K$ diagram. Although the exact location of the giant sequence for the DENIS filter set has not yet been established, 0.4 magnitudes is the typical separation between the dwarf and giant sequences in these filters at this spectral type (Bessell \& Brett 1988). With DENIS data alone, however, such a photometric criterion can only be used for stars which are at least two magnitudes brighter than the detection limit. In general follow-up photometry or spectroscopy is thus essential to separate giants from dwarfs.

\subsubsection{Spectral type indicators}

Jones et al. (1994) have shown that luminosity $(L)$ and/or effective temperature $\left(T_{\text {eff }}\right)$ information can be obtained for late-type dwarfs using features in their infrared spec- tra. In particular, the strength of $\mathrm{H}_{2} \mathrm{O}$ (as measured by the slope of the pseudo-continuum in regions of stellar $\mathrm{H}_{2} \mathrm{O}$ absorption) is a sensitive measure of the $T_{\text {eff }}$ of the stellar photosphere. For main sequence dwarfs, therefore, a relationship between $L$ and the strength of $\mathrm{H}_{2} \mathrm{O}$ features can obviously be obtained, since there is essentially a one-to-one mapping between $L$ and $T_{\text {eff }}$.

The same is also largely true for brown dwarfs. As they age they slide along an extension of the main sequence in an $H-R$ diagram (see e.g. D'Antona \& Mazzitelli 1985; Burrows et al. 1989; Burrows et al. 1997). The luminosity spread in this main sequence "extension" due to mass and age differences is $\sim 1$ magnitude, which is similar to that seen due to metallicity variation in lowmass stars (e.g. Tinney et al. 1995). So even in the absence of parallaxes or atmospheric models, spectral features can provide luminosity information for brown dwarfs, as they do for low-mass stars.

We therefore use the slope of a straight-line fit to each $F_{\nu}$ spectrum in the wavelength ranges $1.51-1.57 \mu \mathrm{m}$ and $2.08-2.16 \mu \mathrm{m}$ to define two $\mathrm{H}_{2} \mathrm{O}$ indices. These wavelength regions were chosen because they are dominated by $\mathrm{H}_{2} \mathrm{O}$ absorption, and because they lie wholly within single echelle orders in our IRIS spectra. The indices are presented for each program object in Table 2, and for each comparison object in Table 3 . Also included in Table 3 are the corresponding indices for the objects GD 165B (Jones et al. 1994) and Gl229B (Geballe et al. 1996). The quoted uncertainties are those produced by the least-squares fitting procedure. In the two cases where repeated observations are available (DENIS-P J0205-1159 and 


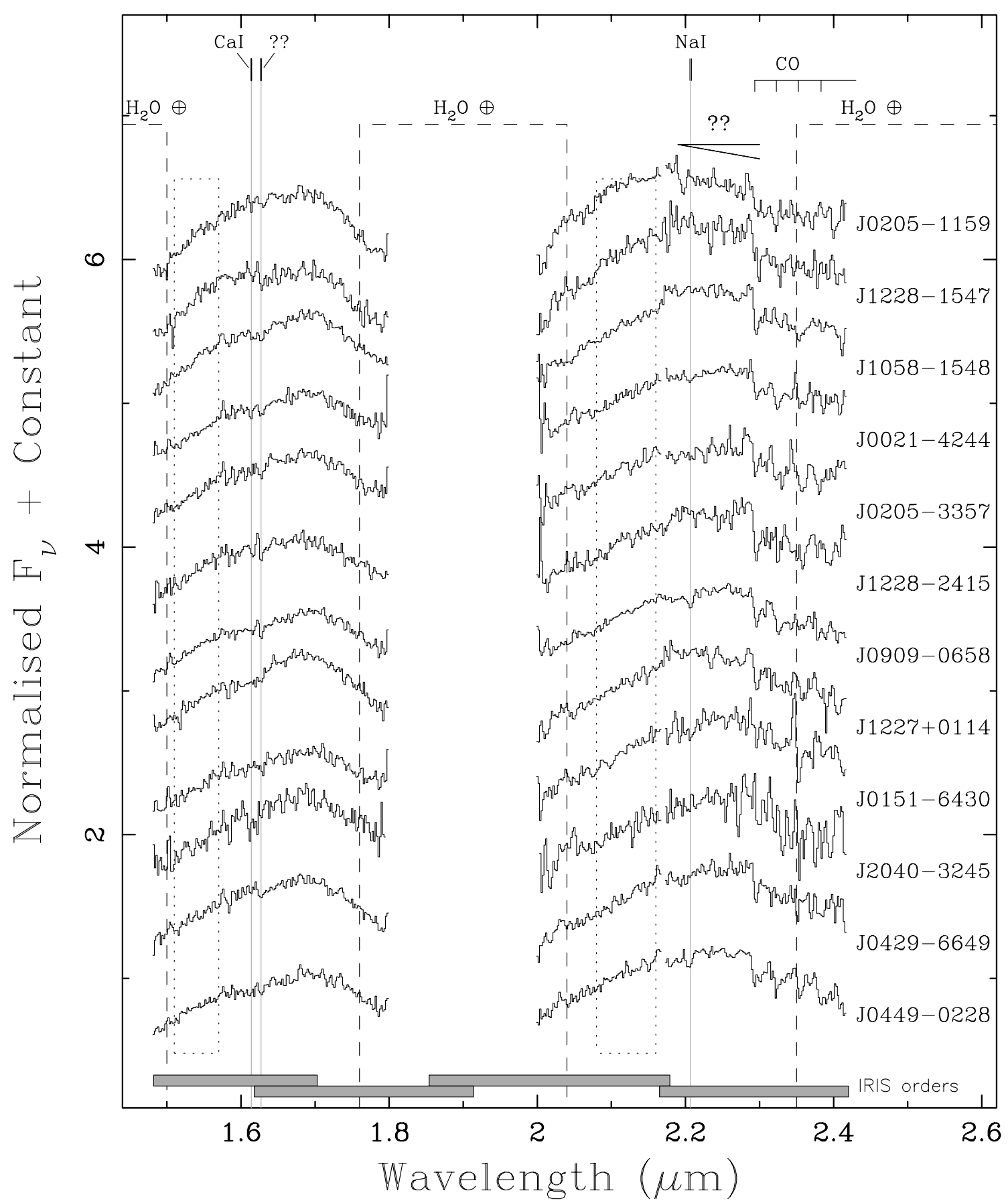

Fig. 7. IRIS HK echelle spectra for representative DENIS dwarfs from Table 2. All spectra have been normalized by their flux integrated between 2.09 and $2.11 \mu \mathrm{m}$, and offset by steps of 0.5 from the preceeding object. Objects are shown ordered by the estimated $M_{K}$ derived as described in the text. The dashed lines show the wavelength ranges where terrestrial $\mathrm{H}_{2} \mathrm{O}$ absorption make the spectra unreliable. The dotted boxes show the wavelength ranges over which the $\mathrm{H}_{2} \mathrm{O}$ indices described in Sect. 2.4.2 were measured

J1228-1547) the measured indices are consistent within the derived uncertainties.

Figures $11 \mathrm{~b}$ and $\mathrm{d}$ show these indices plotted as a function of $I-J$ colour, while Figs. 11c and e show them plotted as a function of $M_{K}$. The $H$-band $(1.51-1.57 \mu \mathrm{m})$ $\mathrm{H}_{2} \mathrm{O}$ index can be seen to show a smooth dependence on $L$ and/or $T_{\text {eff }}$. The $K$-band index $(2.08-2.16 \mu \mathrm{m})$, on the other hand, shows a marked turnover somewhere between effective temperatures corresponding to GD 165B $(1900 \pm 100 \mathrm{~K}$; Kirkpatrick et al. 1998) and those corresponding to the low-temperature $(\sim 1000 \mathrm{~K})$ brown dwarf Gl229B. This turnover is almost certainly due to the onset of $\mathrm{CH}_{4}$ absorption in the $K$-band for temperatures below $1500 \mathrm{~K}$ - this is clearly seen in Gl229B in Fig. 9. 


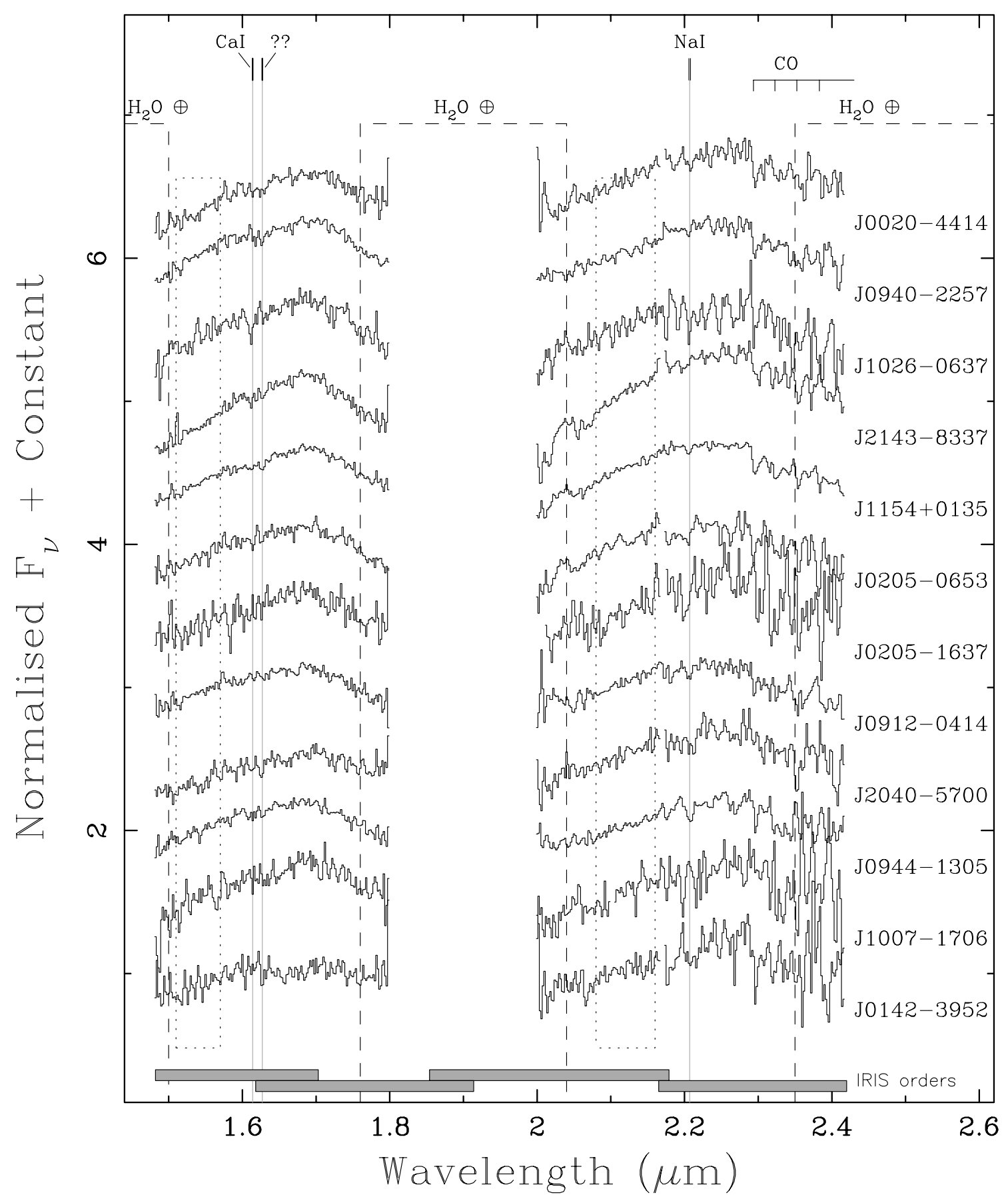

Fig. 8. IRIS HK echelle spectra (continued). See Fig. 7 caption

The $\mathrm{H}_{2} \mathrm{O}$ indices for all observations of the Mini-survey objects are also plotted in Figs. 11b and d as a function of their DENIS $I-J$ colour. We can immediately see that none of the DENIS objects show $\mathrm{H}_{2} \mathrm{O}$ indices indicating them to be as cool as Gl229B. This is not surprising, since the $\mathrm{CH}_{4}$ features of $\mathrm{Gl} 229 \mathrm{~B}$ are distinctive, and would be immediately apparent in the spectra. However, it is comforting to see that the $\mathrm{H}_{2} \mathrm{O}$ indices confirm this expectation.
In order to use these $\mathrm{H}_{2} \mathrm{O}$ indices to estimate absolute magnitudes for the Mini-survey objects, we need to establish a calibration. Figure 11 clearly shows that for objects as faint, or fainter than, GD 165B such a calibration is, at present, poorly constrained. As the photometric distances for the coolest DENIS objects are only $\lesssim 25$ pc, parallax measurement for these objects will be straightforward. Further refinement of the $\mathrm{H}_{2} \mathrm{O}$-index-to- $M_{K}$ calibrations can therefore be expected in the future. For the time 


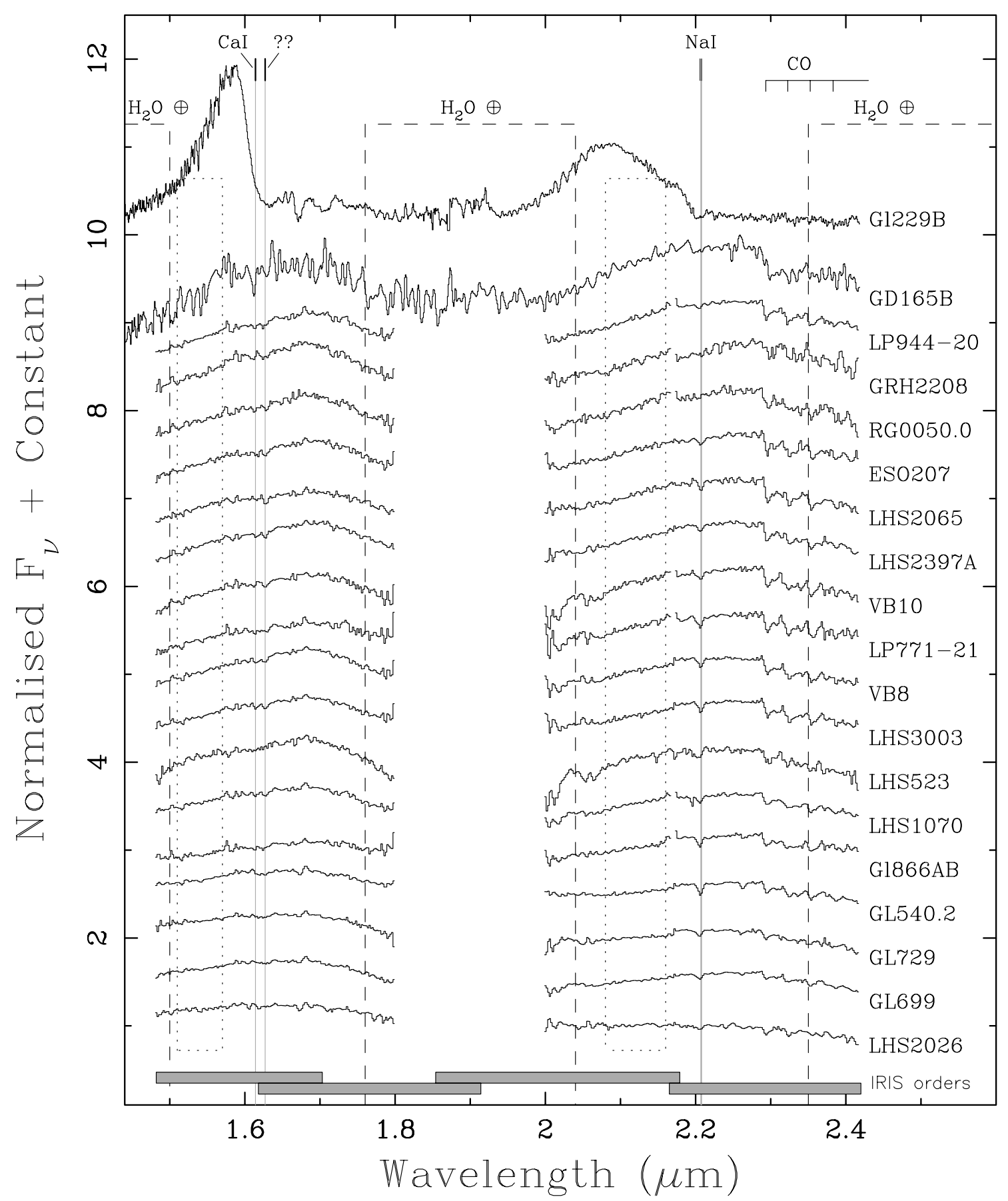

Fig. 9. IRIS HK echelle spectra of the template dwarf objects listed in Table 3. All spectra have been normalized by their flux integrated between 2.09 and $2.11 \mu \mathrm{m}$, and offset by steps of 0.5 from the preceeding object, with the exception of of Gl229B which has been offset by 1.5. The dashed lines show the wavelength ranges where terrestrial $\mathrm{H}_{2} \mathrm{O}$ absorption make the spectra unreliable. The dotted boxes show the wavelength ranges over which the $\mathrm{H}_{2} \mathrm{O}$ indices described in Sect. 2.4.2 were measured

being, however, we adopt a minimal calibration consisting of two linear fits to the available data, with a break at $M_{K}=11$. The adopted fits are shown in Figs. 11c and e. As a result of the degeneracy in the $2.08-2.16 \mu \mathrm{m} \mathrm{H}_{2} \mathrm{O}$ index it is clearly not useful for estimating luminosities for our sample. We have therefore derived $M_{K}$ estimates (which are shown in Table 2) using the $1.51-1.57 \mu \mathrm{m} \mathrm{H}_{2} \mathrm{O}$ index alone. The uncertainties quoted in these estimated luminosities are based on the measured uncertainties in the $\mathrm{H}_{2} \mathrm{O}$ indices propagated through the $\mathrm{H}_{2} \mathrm{O}$-index-to$M_{K}$ calibration, added in quadrature to the uncertainty in the calibration as derived from the residuals about 
Table 3. Template object spectral classifiers

\begin{tabular}{|c|c|c|c|c|c|c|c|}
\hline Object & $I-J$ & $M_{K}$ & Spectral & \multicolumn{2}{|c|}{$\mathrm{H}_{2} \mathrm{O}$ Indices } & \multirow{2}{*}{$\frac{\mathrm{CO}^{b}}{\text { Index }}$} & \multirow[t]{2}{*}{ References $^{c}$} \\
\hline & & & Type $^{a}$ & $1.51-1.57$ & $2.08-2.16$ & & \\
\hline & \multicolumn{2}{|c|}{ (a) M-giant templates } & & & & & \\
\hline BS681 & - & - & - & $7.44 \pm 0.31$ & $4.24 \pm 0.15$ & 1.243 & \\
\hline BS8636 & - & - & - & $1.49 \pm 0.20$ & $-0.16 \pm 0.11$ & 1.278 & \\
\hline BS1492 & - & - & - & $1.73 \pm 0.39$ & $0.67 \pm 0.09$ & 1.277 & \\
\hline \multirow[t]{2}{*}{ BS8637 } & - & - & - & $0.58 \pm 0.20$ & $-0.48 \pm 0.12$ & 1.242 & \\
\hline & \multicolumn{5}{|c|}{ (b) M-dwarf and Brown dwarf templates } & & \\
\hline Gl229B & $5.80 \pm 0.10$ & $15.6 \pm 0.10$ & - & $17.2 \pm 0.5$ & $-6.71 \pm 0.17$ & 1.159 & 11,7 \\
\hline GD165B & $3.37 \pm 0.10$ & $11.7 \pm 0.20$ & $\geq \mathrm{M} 10 \mathrm{~V}$ & $6.90 \pm 0.97$ & $2.56 \pm 0.15$ & 1.284 & 9,10 \\
\hline LP944-20 & $3.39 \pm 0.07$ & $11.10 \pm 0.06$ & $\geq \mathrm{M} 9 \mathrm{~V}$ & $3.66 \pm 0.16$ & $2.57 \pm 0.13$ & 1.134 & 1,2 \\
\hline GRH2208 & - & - & - & $3.28 \pm 0.28$ & $2.63 \pm 0.21$ & 1.062 & 1,8 \\
\hline RG0050.0 & $3.13 \pm 0.07$ & $39 \pm 0.48$ & M8V & $2.78 \pm 0.25$ & $2.80 \pm 0.20$ & 1.159 & 1,3 \\
\hline ESO207-61 & $2.83 \pm 0.05$ & $10.84 \pm 0.20$ & - & $2.96 \pm 0.18$ & $2.08 \pm 0.16$ & 1.127 & 1 , \\
\hline LHS2065 & $3.20 \pm 0.05$ & $10.34 \pm 0.05$ & M9V & $2.85 \pm 0.20$ & $2.38 \pm 0.14$ & 1.176 & 4,5 \\
\hline LHS2397A & $3.00 \pm 0.05$ & $9.80 \pm 0.15$ & M8V & $2.93 \pm 0.17$ & $1.89 \pm 0.10$ & 1.154 & $1,4,5$ \\
\hline VB10 & $2.90 \pm 0.05$ & $9.90 \pm 0.07$ & M8V & $2.97 \pm 0.21$ & $2.25 \pm 0.18$ & 1.155 & 4,5 \\
\hline LP771-21 & $2.82 \pm 0.07$ & $10.42 \pm 0.20$ & M8V & $2.69 \pm 0.20$ & $2.03 \pm 0.16$ & 1.121 & 1,2 \\
\hline VB8 & $2.47 \pm 0.05$ & $9.78 \pm 0.05$ & M7V & $2.41 \pm 0.16$ & $1.68 \pm 0.13$ & 1.145 & 4 \\
\hline LHS3003 & $2.60 \pm 0.05$ & $10.08 \pm 0.08$ & M7V & $2.33 \pm 0.14$ & $1.58 \pm 0.13$ & 1.133 & 1 , \\
\hline LHS523 & $2.27 \pm 0.05$ & $9.47 \pm 0.07$ & - & $2.16 \pm 0.24$ & $1.77 \pm 0.21$ & 1.123 & 7, \\
\hline LHS1070 & $2.46 \pm 0.05$ & $8.81 \pm 0.10$ & - & $1.90 \pm 0.14$ & $1.64 \pm 0.08$ & 1.097 & 7,5 \\
\hline GL866AB & $2.12 \pm 0.05$ & $7.90 \pm 0.05$ & - & $1.41 \pm 0.19$ & $1.32 \pm 0.11$ & 1.083 & 7,5 \\
\hline GL540.2 & $1.76 \pm 0.05$ & - & - & $1.12 \pm 0.11$ & $0.79 \pm 0.08$ & 1.082 & 7,5 \\
\hline GL699 & $1.50 \pm 0.05$ & $8.06 \pm 0.05$ & - & $0.85 \pm 0.09$ & $0.73 \pm 0.07$ & 1.059 & 7, \\
\hline GL729 & $1.48 \pm 0.05$ & $8.02 \pm 0.05$ & - & $0.61 \pm 0.10$ & $0.53 \pm 0.07$ & 1.080 & 7,5 \\
\hline LHS2026 & $2.25 \pm 0.05$ & $9.71 \pm 0.05$ & - & $0.42 \pm 0.14$ & $0.26 \pm 0.12$ & 1.045 & 7,5 \\
\hline
\end{tabular}

Notes:

$a$ - Spectral types are due to Kirkpatrick et al. (1997b) and Kirkpatrick et al. (1995),

$b$ - uncertainty in $\mathrm{CO}$ ratio $< \pm 0.005$.

$c$ - References for photometry and astrometry: (1) Tinney 1996; (2) Kirkpatrick et al. 1997b; (3) Reid \& Gilmore 1981; (4) Monet et al. 1992; (5) Leggett 1992; (6) Ruiz et al. 1991; (7) Gliese \& Jahreiss 1991; Gliese 1969; (8) Gilmore et al. 1985; (9) C.Dahn, private communication, see Sect. 3.2; (10) Tinney et al. 1993; (12) Matthews et al. 1996.

the calibration fit. Objects with $\mathrm{H}_{2} \mathrm{O}$ indices outside the range provided for by our calibration are denoted in the table as having $M_{K}<8$.

\section{Discussion}

\subsection{A large sample of very low mass stars and brown dwarfs}

Our infrared spectroscopy, then, indicates that the source list contains 17 objects with $M_{K} \geq 11$, and 3 objects with $M_{K} \geq 11.5$. Alternatively, we can consider the $1.51-$ $1.57 \mu \mathrm{m} \mathrm{H}_{2} \mathrm{O}$ index as an estimator of spectral type, in which case Table 3 indicates that objects M8V and later will have a $\mathrm{H}_{2} \mathrm{O}$ index $\gtrsim 2.5$. There are 19 such objects in our sample.

Thus the analysis of only a small fraction of the existing DENIS data has significantly increased (i.e. by $\approx 60 \%$ - Kirkpatrick 1998) the known inventory of very late dwarfs. Seven of those have spectral type of M9-M9.5V or later (based on their $1.51-1.57 \mu \mathrm{m} \mathrm{H}_{2} \mathrm{O}>4$ ), where eight were previously known (Kirkpatrick 1998). These very late objects are DENIS-P J0205-3357, J1228-2415, J00214244, J2146-2153, J1058-1548, J1228-1547 and J02051159.

We have obtained excellent infrared spectra for all except DENIS-P J2146-2153 (Fig. 7) and they are very similar to those of the M9V templates. The spectrum of DENIS-P J2146-2153 (not shown in Fig. 7) is noisier, but also consistent with dwarfs cooler than M9V. Exact typing on the current $M$ system will require optical spectra, but all are certainly new M9-M9.5 dwarfs. At least half (DENIS-P J0205-1159, DENIS-P J1228-1547, J10581548 and J0021-4244) have photometric distances placing them inside $25 \mathrm{pc}$, and are candidates for inclusion in the Catalogue of Nearby Stars.

Optical spectra have been obtained for a number of the Mini-survey sources, and are discussed in more detail by Tinney et al. (1998, hereafter TDFA), who find that optical spectral typing of those objects produce a $T_{\text {eff }}$ 


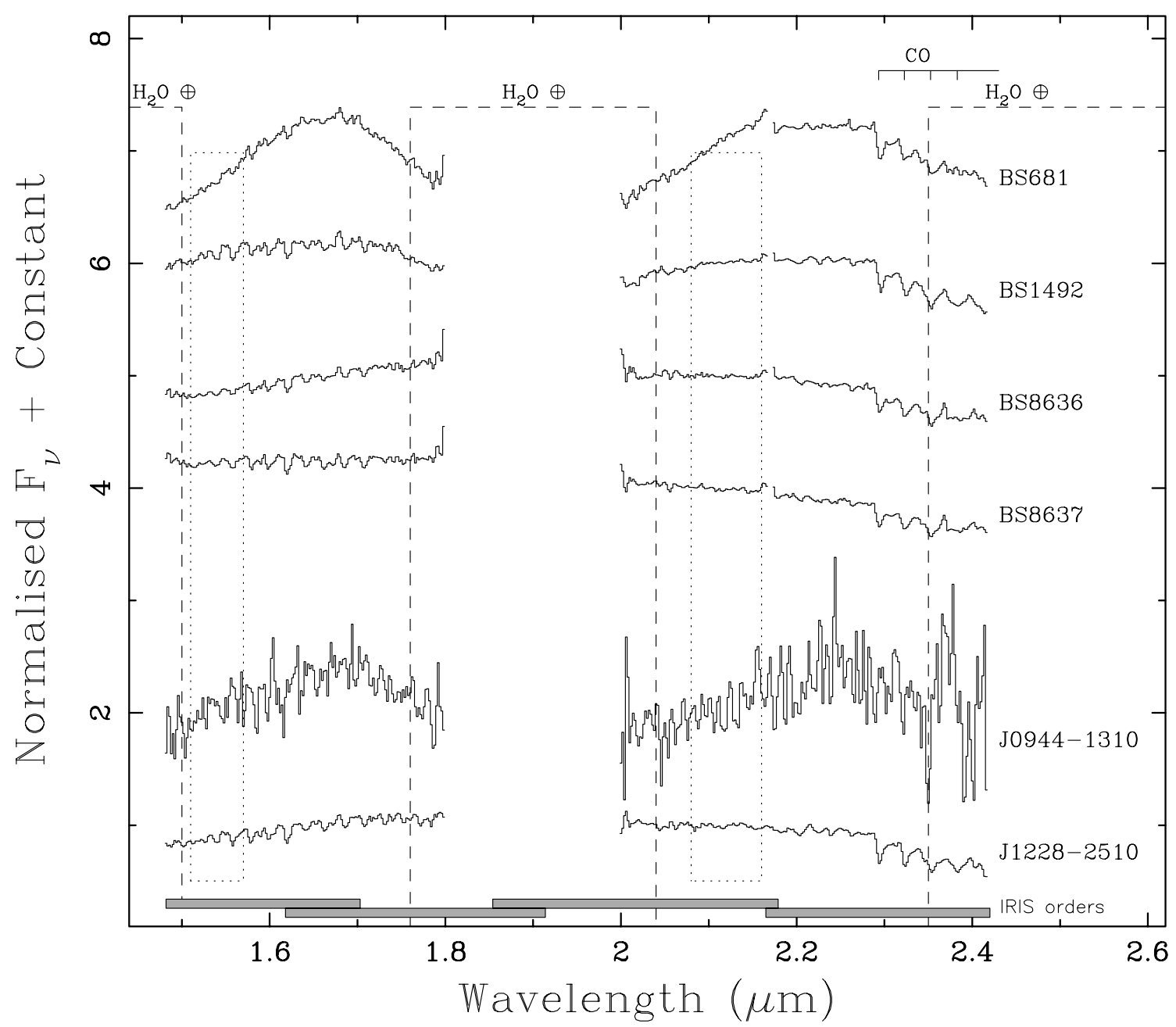

Fig. 10. IRIS HK echelle spectra of the template giants (cf. Table 3), and the two DENIS objects classified as giants in Sect. 2.4.1. All spectra have been normalized by their flux integrated between 2.09 and $2.11 \mu \mathrm{m}$, and offset by steps of 1.0 from the preceeding object, with an added step of 1.0 between the template and target stars. The dashed lines show the wavelength ranges where terrestrial $\mathrm{H}_{2} \mathrm{O}$ absorption make the spectra unreliable. The dotted boxes show the wavelength ranges over which the $\mathrm{H}_{2}$ indices described in Sect. 2.4.2 were measured

ordering consistent with that produced by the $\mathrm{H}_{2} \mathrm{O}$ indices described above.

The reddest three objects detected in the DENIS Minisurvey are DENIS-P J0205-1159, J1058-1548 and J12281547. Both their DENIS colours, and their infrared spectra indicate they are as late in type as GD 165B, or later. They are discussed in more detail by Delfosse et al. (1997, hereafter D97), TDFA, Martín et al. (1997, hereafter MBDF) and Tinney et al. (1997, hereafter TDF). Briefly, the optical (TDFA,TDF) and infrared (D97) spectra of DENISP J1058-1548 are very similar to those of GD 165B. It must have the same effective temperature, for which the current best estimate is $1900 \pm 100 \mathrm{~K}$, based on dusty atmospheric models (Kirkpatrick et al. 1998). The visible and infrared spectra of DENIS-P J0205-1159 and J12281547 are both cooler than GD 165B. DENIS-P J0205-1159 is the coolest isolated dwarf identified to date. DENISP J1228-1547 has an effective temperature intermediate between those of GD 165B and J0205-1159. It has been demonstrated to be a brown dwarf by both MBDF and TDF. They have shown that lithium is abundant in the photosphere of this fully convective object, and therefore that its mass has to be lower than the lithium burning threshold of $\sim 0.06$ solar masses. Taken together, the data and the available models therefore imply that DENISP J1228-1547 is a brown dwarf. DENIS-P J0205-1159 has a high probability of being a brown dwarf, because of its very low effective temperature. The effective temperature scale below $2000 \mathrm{~K}$ is however sufficiently uncertain that it could also be a very low mass star. DENIS-P J1058-1548 may also be a brown dwarf, but we consider its status like GD 165B - more uncertain.

The $\mathrm{TiO}$ and VO bands which constitute the defining characteristic of the $\mathrm{M}$ spectral class are absent, or very weak, in the optical spectrum of these very cool dwarfs (TDF, MBDF). This is almost certainly due to 


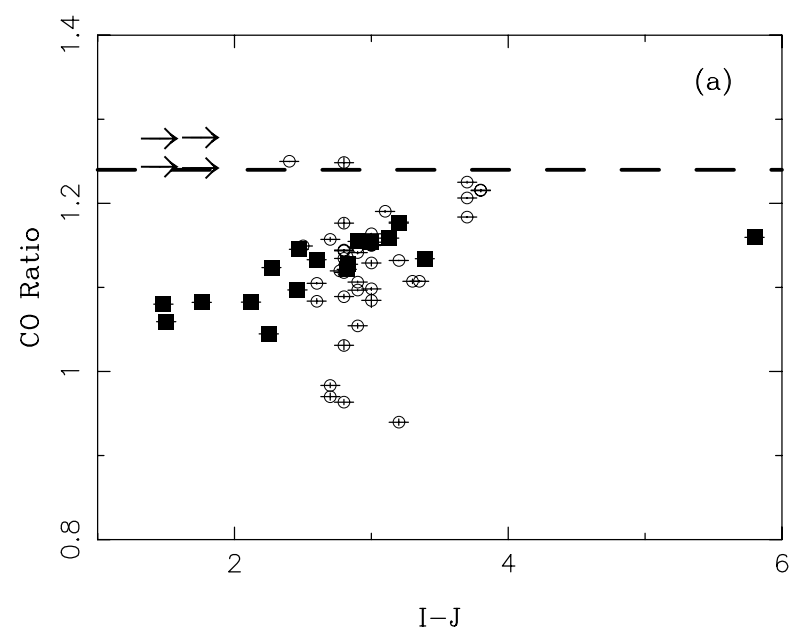

im Template M-dwarfs

$\rightarrow$ Template $\mathrm{M}$-giants

- Target objects


Fig. 11. Infrared Spectral Classification for VLM stars and brown dwarfs. Panel a) shows the CO index (as defined in Sect. 2.4.1) as a function of $I-J$ colours for both our template dwarfs and giants, and our target objects. Panels b) and d) show the $\mathrm{H}_{2} \mathrm{O}$ indices at $(1.51-1.57) \mu \mathrm{m}$ and $(2.08-2.18) \mu \mathrm{m}$ (respectively) as a function of $I-J$ colour for both template and target dwarfs. Panels c) and e) show the same indices as a function of $M_{K}$ for the template dwarfs with trigonometric parallaxes. The solid lines in panels c) and e) are two component linear fits used to estimate $M_{K}$ as described in the text 
depletion of heavy elements in their cool photospheres by the formation of dust grains - in particular solid VO and perovskite $\left(\mathrm{CaTiO}_{3}\right)$ (Sharp \& Huebner 1990; Allard 1998). Kirkpatrick (1998) has advocated that a new spectral class should be defined to describe these spectra. Both Kirkpatrick et al. (1998) and MBDF have proposed the designation "L" for this new spectral class. To date, five published objects can be considered L-type dwarfs: GD 165B, Kelu-1 (Ruiz et al. 1997), DENIS-P J02051159, J1058-1548 and J1228-1547. The optical spectrum of Gl 229B shows no $\mathrm{TiO}$ or VO absorption (Oppenheimer et al. 1998). However, it also shows none of the molecular features seen in the remaining L-type dwarfs, so whether or not it should be considered an L-dwarf remains problematic. Though the existing sample is too small to define a detailed typing scheme, work on this is progressing (Kirkpatrick \& Reid 1998).

The on-going processing of the DENIS survey should eventually provide a sample of about 300 L-dwarfs. Already the present Mini-survey has produced the largest sample of very low mass dwarfs, and its follow-up programs (astrometry and visible spectroscopy) will provide much needed observational constraints for low mass dwarf interior and atmospheric models.

\subsection{The space density of GD165B-like L-dwarfs}

Any estimate of the space density of L-dwarfs will, of necessity, be very uncertain. This is due to both the large uncertainties in the colour-luminosity relations required to evaluate the distances to objects, and to the small number of objects (3) detected in an homogenous survey. On the observational side only one L-dwarf, GD 165B, has a known parallax and absolute magnitudes. This is of course insufficient to properly calibrate the necessary color-luminosity relations. On the theoretical side, the most recent tracks which consistently combine internal structure and atmospheric models (e.g. Chabrier \& Baraffe 1997) still rely on non-dusty model atmospheres. The influence of dust formation on the emerging spectra of extremely cool dwarf is now clearly established (Jones \& Tsuji 1997; Allard 1998) and dusty model atmospheres are needed to provide an acceptable description of the spectra of L-dwarfs.

We therefore adopt the absolute magnitude of GD $165 \mathrm{~B}\left(M_{K}=11.7 \pm 0.2, M_{J}=13.3 \pm 0.2, M_{I}=16.7 \pm 0.2\right.$, C. Dahn, private communication), as representive of all three DENIS L-dwarfs, in spite of their slightly different colours and spectra. Under these assumptions, the generalised $1 / \mathrm{V}_{\max }$ estimator (Schmidt 1968; Felten 1976; Stobie et al. 1989) of the space density is $\Phi_{1}=0.02 \pm$ $0.01 \mathrm{pc}^{-3}$. As two of these object are cooler than GD 165B and certainly fainter, this value is a firm lower limit on the space density.

If on the other hand we use the $M_{K}$ derived from the slope of the infrared spectra in Sect. 2.4.2 $\left(M_{K}=11.5 \pm 0.5\right.$ for DENIS-P J1058-1548; $M_{K}=12.0 \pm 0.5$ for DENISP J1228-1547 and $M_{K}=12.1 \pm 0.5$ DENIS-P J02051159) we obtain a second estimate of the space density, $\Phi_{2}=0.04 \pm 0.02 \mathrm{pc}^{-3}$. This estimate is also uncertain, as it relies on a spectral shape to luminosity calibration that extrapolates on the absolute $K$ magnitude of GD 165B, which itself is only moderately well determined. We consider that it is more likely to overestimate the true luminosity function than to underestimate it.

In both of the above cases, however, the raw luminosity function will be systematically overestimated. This is because the considerable uncertainty in our absolute magnitudes will bias us to sample larger volumes than we expect - i.e. we have a classical Malmquist bias (Stobie et al. 1989). In this case the classical bias dominates over all other biases because, while our infrared spectra give systematically uncertain $M_{K}$ values, we can be certain that no more luminous dwarfs have been scattered into our GD165B-like sample. We can derive an estimate of the magnitude of this effect using Eq. (12) of Stobie et al., assuming a locally flat luminosity function, and a scatter of 0.5 magnitude (uncertainty on our absolute magnitudes). We find that our raw luminosity function will be systematically overestimated by about a third, giving corrected luminosity function estimates of $\Phi_{1}^{\prime}=0.013 \pm 0.007 \mathrm{pc}^{-3}$ and $\Phi_{2}^{\prime}=0.026 \pm 0.014 \mathrm{pc}^{-3}$.

The field star bolometric luminosity function has a maximum of $\approx 0.02 \mathrm{pc}^{-3} \mathrm{mag}^{-1}$ at $M_{\mathrm{bol}} \approx 10$, and then decreases to a plateau of $\sim 0.005 \mathrm{pc}^{-3} \mathrm{mag}^{-1}$ at $M_{\text {bol }}>11$ (e.g. Kroupa 1995; Tinney 1992). If we assume our GD165B-like luminosity function bin covers approximately $M_{K}=11.5-12.5$ or $M_{\text {bol }}=14.7-15.9$ (Tinney et al. 1993), we derive a lower bound to the bolometric luminosity function at $M_{\text {bol }}=15.3$ of $0.011 \pm$ $0.006 \mathrm{pc}^{-3} \mathrm{mag}^{-1}$, allowing the possibility that the luminosity function may increase through the begining of the brown dwarf domain.

On-going analysis of a larger fraction of the existing DENIS data will soon provide a much larger sample of GD 165B-like brown dwarfs, and considerably reduce the present large statistical uncertainties. It will also efficiently select the handful of cool Gl229B-like brown dwarfs which will be present in the survey. The other dominant uncertainty for the luminosity function in this range is our poor knowledge of the colour-luminosity relation. We are presently addressing this problem by measuring the parallaxes of the objects discussed here, and plan a parallax follow-up of all brown dwarfs found in the full DENIS survey. This will provide both a well defined set of color-luminosity relations, and a luminosity function for L-dwarfs significantly free of the Malmquist-type biases which have plagued the study of more massive stars via photographic plates.

Analysis and follow-up of the DENIS survey data will also have important implications for VLM research, since it will be able to identify a complete distance-limited 
sample of nearby stars for which parallaxes can be easily measured. This will enable us to settle the nearbystar vs photometric luminosity function question once and for all by "superseding" the photometric function with a well sampled and almost "bias-free" nearby star luminosity function.

Acknowledgements. We warmly thank the members of the DENIS consortium whose work made these results possible. We are grateful to Isabelle Baraffe, Gilles Chabrier, France Allard, Davy Kirkpatrick, Neill Reid and Eduardo Martín for useful discussions, and to Hugh Jones and Tom Geballe for providing digital copies of their GD 165B and Gl 229B spectra (respectively). We would also like to thank the technical and astronomical support staff at the AAT for their most professional assistance throughout this observing program. The DENIS project is partly funded by the European Commission through SCIENCE and Human Capital and Mobility grants. It is also supported in France by INSU, the Education Ministry and CNRS, in Germany by the Land of Baden-Würtenberg, in Spain by DGICYT, in Italy by CNR, in Austria by the Fonds zur Förderung der Wissenshaftlichen Forschung and Bundesministerium für Wissenschaft und Forschung, in Brazil by FAPESP, and in Hungary by an OTKA grant and an ESO C\&EE grant. X.D. acknowledges a "Lavoisier" grant of the French ministère des affaires étrangères.

\section{References}

Allard F., 1998, in Brown Dwarfs \& Extra-solar Planets, Rebolo, Martín \& Zapaterio-Osorio (eds.), ASP Conf. 134, 370

Allen D.A., et al., 1993, Proc. Astron. Soc. Aust. 10, 298

Basri G., Marcy G.W., Graham J.R., 1996, ApJ 458, 600

Becklin E.E., Zuckerman B., 1988, Nat 336, 656

Bertin E., Arnouts S., 1996, A\&AS 117, 393

Bessell M.S., Brett J.M., 1988, PASP 100, 1134

Bessell M.S., Castelli F., Plez B., 1998, A\&A 333, 231

Borsenberger J., 1997, in Proceedings of the $2^{\text {nd }}$ DENIS Euroconference The impact of large scale near-infrared surveys, Garzon F., Epchtein N., Omont A., Burton W.B., Persi P. (eds.). Kluwer Ac. Publishers, Dordrecht, p. 181

Burrows A., Marley M., Hubbard W.B., et al., 1997, ApJ 491, 856

Burrows A., Hubbard W.B., Lunine J.I., 1989, ApJ 345, 939

Carter B.S., Meadows V.S., 1995, MNRAS 276, 734

Chabrier G., Baraffe I., 1997, A\&A 327, 1039

Copet E., et al., 1998, A\&A (in press)

D'Antona F., Mazzitelli I., 1985, ApJ 296, 502

Delfosse X., et al., 1997, A\&A 327, L25 (D97)

Deul E., et al., 1995, in Euroconference on Near Infrared Sky Surveys, Persi P., Burton B., Epchtein N., \& Omont A. (eds.), Memor. della Soc. Astron. Ital. 66, 3, p. 549

Epchtein N., et al., 1994, in Science with Astronomical NearInfrared Sky Surveys, Epchtein N., Omont A., Burton B. \& Persi P. (eds.). Kluwer: Dordrecht, p. 3
Epchtein N., 1997, in Proceedings of the $2^{\text {nd }}$ DENIS Euroconference The impact of large scale near-infrared surveys, Garzon F., Epchtein N., Omont A., Burton W.B., Persi P. (eds.). Kluwer Ac. Publishers, Dordrecht, p. 15

Felten J.E., 1976, ApJ 207, 700

Geballe T.R., Kulkarni S.R., Woodward C.E., Sloan G.C., 1996, ApJ 467, L101

Gilmore G., Reid N., Hewett P., 1985, MNRAS 213, 257

Gliese W., 1969, Veroffentlichungen des Astronomischen Rechen Instituts, Heidelberg, p. 22

Gliese W., Jahreiss H., 1991, in The Astronomical Data Center CD-ROM: Selected Astronomical Catalogs, Vol. 1, L.E. Brotzmann, S.E. Gesser (eds.), NASA/ADC, Greenbelt, MD, U.S.A.

Hawkins M.R.S., Bessell M.S., 1988, MNRAS 234, 177

Jones H.R.A., Longmore A.J., Jameson R.F., Mountain C.M., 1994, MNRAS 267, 413

Jones H.R.A., Tsuji T., 1997, ApJ 380, 39

Kirkpatrick J.D., 1998, in "Brown Dwarf and Extrasolar Planets Workshop", Rebolo, Martín, Zapatero Osorio (eds.), ASP Conf. Ser. 134, 405

Kirkpatrick J.D., Allard F., Bida T., et al., 1998, ApJ (submitted)

Kirkpatrick J.D., Beichman C.A., Skrutskie M.F., 1997a, ApJ 476,311

Kirkpatrick J.D., Henry T.J., Irwin M.J., 1997b, AJ 113, 1421

Kirkpatrick J.D., Reid I.N., 1998 (in preparation)

Kroupa P., 1995, ApJ 453, 358

Lang K.R., 1991, Astrophysical Data, Planets and Stars. Springer-verlag: New York

Leggett S.K., 1992, ApJS 82, 351

Martín E.L., Zapatero Osorio M.R., Rebolo R., 1998, in "Brown Dwarf and Extrasolar Planets Workshop", Rebolo, Martín, Zapatero Osorio (eds.), ASP Conf. Ser. 134, 507

Martín E.L., Basri G., Delfosse X., Forveille T., 1997, A\&A 327, L29 (MBDF)

Matthews K., Nakajima T., Kulkarni S.R., Oppenheimer B.R., 1996, AJ 112, 1678

Monet D.G., Dahn C.C., Vrba F.J., et al., 1992, AJ 103, 638

Nakajima T., Oppenheimer B.R., Kulkarni S.R., Golimovski D.A., Matthews K., Durrance S.T., 1995, Nat 378, 463

Oppenheimer B.R., Kulkarni S.R., Matthews K., van Kerkwijk M.H., 1998, ApJ (submitted, astro-ph/9802299)

Rebolo R., Zapatero Osorio M.R., Martín E.L., 1995, Nat 377, 129

Reid I.N., Gilmore G., 1981, MNRAS 196, 15p

Reid I.N., Gilmore G., 1984, MNRAS 206, 19

Ruiz M.T., Takamiya M.Y., Roth M., 1991, ApJ 367, L61

Ruiz M.T., Leggett S.K., Allard F., 1997, ApJ 491, L107

Schmidt M., 1968, ApJ 151, 393

Sharp C.M., Huebner W.F., 1990, ApJS 72, 417

Shortridge K., 1993, in Astronomical Data Analysis Software and Systems II, ASP Conf. Ser. 52, 219

Stobie R.S., Ishida K., Peacock J.A., 1989, MNRAS 238, 709

Tinney C.G., 1992, ApJ 414, 279

Tinney C.G, 1996, MNRAS 281, 644

Tinney C.G., Mould J.R., Reid I.N., 1993, AJ 105, 1045

Tinney C.G., Reid I.N., Gizis J., Mould J.R., 1995, AJ 110, 3014

Tinney C.G, Delfosse X., Forveille T., 1997, ApJL 490, L95 (TDF) 
Tinney C.G, Delfosse X., Forveille T., Allard F., 1998, A\&A (submitted) (TDFA)

Totten E.J., Irwin M.J., 1998, MNRAS 291, 1

Tsuji T., Ohnaka K., Aoki W., 1995, in "The Bottom of the Main Sequence and Beyond", Tinney C.G. (ed.). SpringerVerlag: Heidelberg, p. 45

Tsuji T., Ohnaka K., Aoki W., 1996a, A\&A 305, L1
Tsuji T., Ohnaka K., Aoki W., Nakajima T., 1996b, A\&A 308, L29

Zapatero Osorio M.R., Rebolo R., Martín E.L., 1997, A\&A 317,164

Zapatero Osorio M.R., Rebolo R., Martín E.L., et al., 1998, in "Brown Dwarf and Extrasolar Planets Workshop", Rebolo, Martín, Zapatero Osorio (eds.), ASP Conf. Ser. 134, p. 51 